\title{
Posicionamento das farmácias e a logística reversa no controle dos medicamentos em desuso
}

\author{
André Luiz Emmel Silva', Jorge André Ribas Moraes², Adriel Rehbein³, \\ Daniel Bencke ${ }^{4}$, Guilherme Gerhard ${ }^{5}$ \\ 1, 2, 3, 4, 5Universidade de Santa Cruz do Sul, RS, Brasil
}

\begin{abstract}
Resumo
O tema "Logística Reversa" vem sendo discutido e incentivado no meio empresarial com maior ênfase devido ao crescimento da conscientização ambiental. Ele trata da importância de reaproveitar, reciclar e reutilizar, no intuito de tornar as empresas agentes de uma maior consciência de sua importância enquanto agentes de mudanças de atitudes para a sociedade como um todo, elevando o seus cuidados quanto à preservação do meio ambiente e, sendo assim, entendido pelos stakeholders como um diferencial competitivo, colocando-as em diferentes posições frente aos concorrentes. Através de uma pesquisa bibliográfica sobre descarte de medicamentos em desuso, juntamente com um estudo de caso em farmácias, foi possível analisar a situação atual sobre o cumprimento e a conscientização existente em relação às normativas relacionadas ao assunto. A metodologia utilizada baseou-se em entrevistas estruturadas enfatizando aspectos relacionados à conduta de descarte, nível de orientação e aderência às normas regulamentadoras. Foram entrevistadas 50 farmácias, verificando-se que apesar de todas estarem implementando um programa de destinação, poucas entendem sobre a destinação ou sabem o que acontece com os resíduos.
\end{abstract}

Palavras-chave: Impacto ambiental, Logística Reversa; Descarte de Medicamentos.

\begin{abstract}
The theme Reverse Logistics has been discussed and encouraged in the business with greater emphasis due to the growth of environmental awareness. It deals with the importance of recycling, recycle and reuse in order to make companies agents and greater awareness of their importance as agents of change in attitudes to society as a whole raising their care about the preservation of the environment and being well understood by stakeholders as a competitive, placing them in different positions against competitors. Through a literature search on disposal of medicines into disuse, along with a case study in pharmacy, it was possible to analyze the current situation on compliance and awareness regarding existing regulations related to the subject. The methodology used was based on structured interviews, emphasizing issues related to the conduct of disposal, level of guidance and adherence to regulatory standards. 50 pharmacies were interviewed, verifying that despite all are implementing a program allocation, few understand about the destination or know what happens to the waste.
\end{abstract}

Keywords: Environmental impact; Reverse Logistics; Disposal of Medicines 


\section{INTRODUÇÃO}

O crescimento exponencial da população mundial, acompanhado da evolução tecnológica vivenciada pela esfera industrial, fez surgir uma infinidade de produtos nos últimos anos. Muitos são produzidos de acordo com a necessidade e preferência de cada cliente. O tempo de vida útil dos produtos também está cada vez mais curto (ROGERS E TIBBEN-LEMBKE, 2001; LEITE, 2009), fazendo com que sejam rapidamente substituídos por outros mais práticos e com tecnologias mais eficazes. Com crescimento extraordinário na elaboração de novos produtos, e ligeiramente na substituição destes, aumenta-se, consequentemente, a quantidade de resíduo gerado. A produção de resíduos faz parte do cotidiano do ser humano (KEMERICH,2013), e o seu equacionamento torna-se um dos maiores desafios relacionados ao meio ambiente com que se defronta a sociedade moderna (JERÔNIMO, 2013).

Os medicamentos também seguem a mesma escala, com aumentos significativos da variedade e quantidade produzidas (MARGONATO, 2008). A presença de fármacos, cosméticos e produtos de higiene pessoal tem sido detectada em águas superficiais, subterrâneas, águas para consumo humano, e até mesmo em solos sujeitos à aplicação de lodo de esgoto (MCCLELLAN, 2010).

Com o intuito de reduzir estas consequências e impulsionadas por recentes legislações (STOCK, 2002; RADONJIC, 2007), com destaque para a Política Nacional de Resíduos Sólidos (Lei 12.605 que institui a PNRS), as empresas estão adotando o processo da logística reversa, que tem como objetivo o reaproveitamento e a reciclagem de produtos e materiais, com a reutilização destes na cadeia de valor, evitando uma nova busca por recursos da natureza e permitindo um descarte ambientalmente correto (SRIVASTAVA, 2008; LAMBERT, 2011; ZHANG, 2011).

A logística reversa tem grande importância para o descarte de medicamentos, pois a maioria das pessoas descarta os medicamentos em desuso ou vencidos no lixo ou na rede pública de esgoto (MELO, 2009; RODRIGUES, 2009), o que traz sérias consequências ao meio ambiente e às pessoas próximas do local (BARCELOS, 2011). A implementação de um sistema eficaz de gerenciamento de resíduos provenientes de serviços de saúde e da comunidade contribui para a redução de riscos à saúde imediata e ao meio ambiente.

Sendo assim, a presente pesquisa procura demonstrar como está o descarte de medicamentos em farmácias da região do Vale do Rio Pardo, localizada no Rio Grande do Sul - Brasil e a aplicabilidade da logística reversa neste processo.

\section{I.I Logística}

De acordo com Ballou (2006) a logística envolve todas as operações relacionadas com planejamento e controle de produção, movimentação de materiais, embalagem, armazenagem e expedição, distribuição física, transporte e sistemas de comunicação que, realizadas de modo sincronizado, podem fazer com que as empresas agreguem valor aos serviços oferecidos aos clientes e também oportunizem um diferencial competitivo perante a concorrência.

A logística é constituída por várias etapas, iniciando pelos fornecedores, passando pela fabricação, seguindo para o varejista, até atingir o consumidor final, objetivo principal de toda a cadeia de suprimento. Além do fluxo de materiais, há também o fluxo de dinheiro e informações em todo processo (NOVAES, 2001).

A responsabilidade da logística consiste em disponibilizar as matérias-primas, produtos semi-acabados e produtos acabados, no local onde são solicitados. É por meio desse sistema que os materiais passam pelo processo de produção e são destinados aos consumidores. O objetivo principal da logística é que estes produtos possam satisfazer as necessidades dos clientes pelo menor custo possível (BOWERSOX, 2001).

\section{I.2 LOGÍSTICA ReVERSA}

Logística reversa é uma área relativamente nova para empresas e sociedade em geral, em todo o mundo. Ela é considerada uma área bem específica que se preocupa com reutilização de produtos e materiais, iniciada na coleta dos produtos e peças usadas até o seu processamento, com o intuito de assegurar uma recuperação sustentável (LEITE, 2009; MELO JUNIOR, 2013).

A logística reversa veio para contribuir na redução dos impactos ambientais causados pelo acúmulo de resíduo. A responsabilidade desse resíduo gerado pelas empresas já está deixando de 
ser do governo; legislações ambientais estão gradativamente passando essa responsabilidade para as empresas ou suas cadeias industriais (LEITE, 2009).

O processo da logística reversa é similar ao da logística direta, mas de modo inverso. Ela gera materiais reaproveitados e estes retornam ao processo de fabricação e distribuição. A logística reversa é a responsável pelo retorno de produtos aos fabricantes para o reaproveitamento dos componentes desse produto ou, pelo menos, para que lhes seja dado um destino ambientalmente correto (SRIVASTAVA, 2008; LAMBERT, 2011; ZHANG, 2011).

Consiste de planejamento, implementação e controle do fluxo de matérias-primas, estoque em processo e produtos acabados. A figura 1 mostra o processo iniciando pelo consumidor até o ponto de origem, com o objetivo de recapturar valor ou realizar um descarte adequado, sendo assim, um processo de responsabilidade compartilhada com o ciclo de vida de um produto (CONSELHO EM REVISTA, 2011).

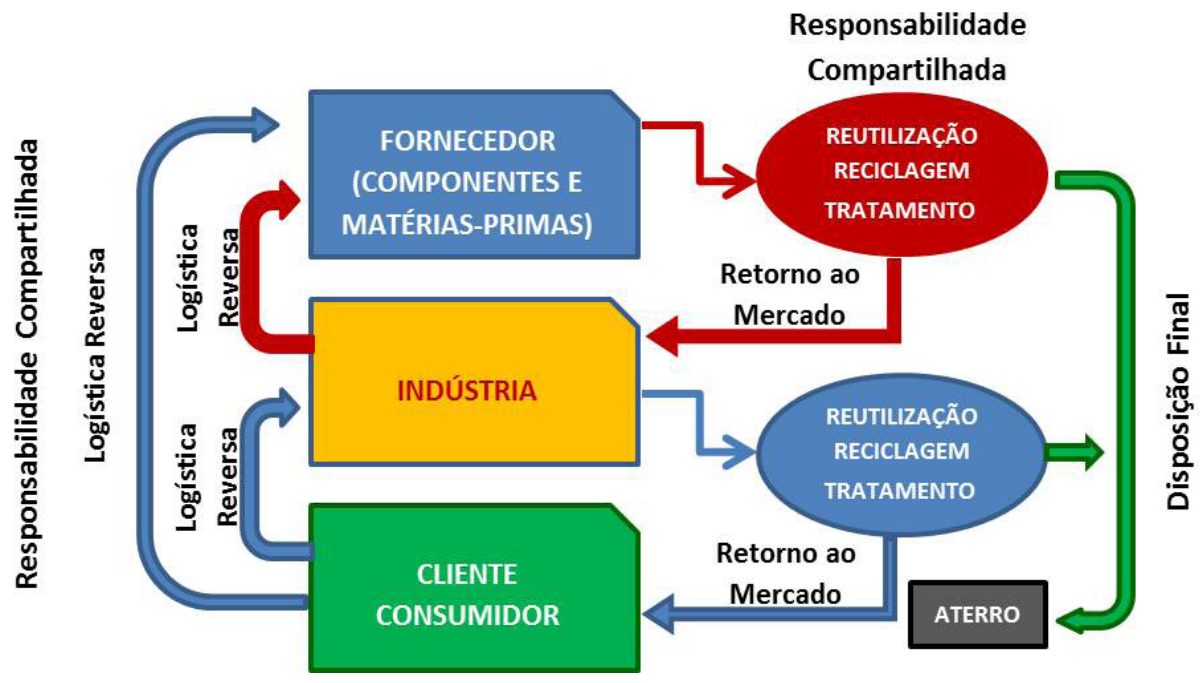

FIGURA 1 - Logística Reversa: Responsabilidade Compartilhada.

Fonte: Conselho em Revista (2011)

No entanto, apesar de sua contante evolução, ainda enfrenta obstáculos e barreiras (RAVI, 2005; LEITE, 2009; DAS, 2012). Falta de sistemas informatizados e integrados, recursos financeiros e recursos de pessoal escassos, menor importância da logística reversa em relação a outras questões e à própria política da empresa, que vê no fluxo reverso apenas custos e não receitas (RAVI, 2005).

O motivo das empresas aderirem ao processo da logística reversa não é somente pela pressão da legislação, mas pelas vantagens que ela pode trazer, como o diferencial competitivo (FERRER, 2000; RAVI, 2005; POKHAREL, 2009). Cada vez é mais significativo o aumento de consciência ecológica dos consumidores, que esperam que as empresas reduzam os impactos negativos de sua atividade ao meio ambiente. As organizações podem aderir a esssa nova demanda que a sociedade já vem fiscalizando e, assim, obterem uma imagem da sua responsabilidade social adequada aos novos padrões de educação ambiental. Esse processo poderá contribuir sobremaneira para que as empresas utilizem dessas informações como marketing, valorizando o seu compromisso com o meio ambiente e com os seus clientes.

Além de proporcionar possíveis oportunidades de economia, alcançadas através da reutilização de matérias-primas, reprocessamento ou reciclagem, a logística reversa pode melhorar a imagem corporativa (SINNECKER, 2007; SILVA, 2012). Representa uma das maiores e mais esquecidas oportunidades de lucratividade para uma empresa. Atualmente, poucas empresas estão fazendo um bom trabalho em abordar esta questão; A maioria está negligenciando sua cadeia de suprimentos de fluxo reverso e estão perdendo oportunidades de melhorar a satisfação e fidelização dos clientes (LAMBERT, 2011; SILVA, 2012). 


\section{I.3 LEGISLAÇÃO RELACIONADA}

O gerenciamento de resíduos no Brasil é abordado em normas gerais ou específicas para determinados setores da cadeia de produção farmacêutica, como na RDC n ${ }^{\circ}$ 306/2004 da ANVISA, Resolução no 358/2005 do CONAMA (Gerenciamento e Destinação Final de RSS) e na RDC n. ${ }^{\circ}$ 17/2010 da ANVISA (Boas Práticas de Fabricação de Medicamentos). Vale ressaltar que estas não estão perfeitamente alinhadas ao novo marco regulatório, a PNRS de 2010. Vários Estados e Municípios também possuem legislação própria, as quais não são claras e, pelo contrário, muitas vezes conflitantes, provocando dúvidas e impossibilitando a adoção de normas práticas e eficazes em todo o País (JOÃO, 2011).

RDC/ANVISA no 306 - Emitida no dia 7 de dezembro de 2004, a Resolução no 306 da Diretoria Colegiada da Agência Nacional de Vigilância Sanitária (ANVISA) contém o Regulamento Técnico sobre o gerenciamento dos Resíduos de Serviços de Saúde (RSS), e como este deve ser observado na área pública e privada em todo território nacional. A não observância da Resolução e sua Regulamentação Técnica caracteriza infração sanitária e submeterá o infrator às penalidades previstas em lei.

A Resolução demonstra a preocupação com aspectos ambientais e de saúde pública, e responsabiliza os serviços de saúde pelo gerenciamento adequado de todos RSS por eles gerados, desde sua geração até sua destinação final. Reduzir quantidades de resíduos perigosos e acidentes ocupacionais, pelo uso inadequado, está entre os resultados pretendidos (FALQUETO, 2010).

O Regulamento Técnico para gerenciamento dos RSS impõe que todo gerador deve ter um Plano de Gerenciamento de Resíduos de Serviços de Saúde (PGRSS) e obedecer aos requisitos citados no Regulamento. O Regulamento contém especificadamente a conceituação e citação dos geradores de resíduos aos quais a regulamentação se aplica, mencionando drogarias e farmácias, inclusive as de manipulação, estabelecimentos de ensino e pesquisa na área de saúde e, também, distribuidores de produtos farmacêuticos (JOÃO, 2011).

RDC ANVISA n ${ }^{\circ}$ 306/04 concentra sua regulamentação no controle dos processos de segregação, acondicionamento, armazenamento, transporte, tratamento e disposição final (RODRIGUES, 2009), mas não oferece para o consumidor final, entendido como aquele que faz uso de medicamentos em sua residência, a mesma riqueza de informações e orientações (BOER, 2011).

Política Nacional de Resíduos Sólidos (PNRS) - Outra normativa, por sinal muito recente, não poderia deixar de ser mencionada aqui. Criada em dois de Agosto de 2010, a Lei $\mathrm{n}^{\circ}$ 12.305, que estabelece a PNRS, traz o assunto "logística reversa" à tona.

Do ponto de vista logístico, o ciclo de vida de um produto não se encerra com a entrega ao cliente. Produtos que se tornam obsoletos, danificados ou não funcionam devem retornar ao ponto de origem para serem adequadamente descartados, reparados ou reaproveitados (GONÇALVES, 2006; SRIVASTAVA, 2008).

A PNRS apresenta a logística reversa como uma das principais ferramentas de gerenciamento de resíduos sólidos a ser aplicado às pessoas jurídicas ou físicas, de direito privado ou público, sejam estas responsáveis, direta ou indiretamente, pela produção dos resíduos. O grande marco conceitual da nova legislação é o conceito de responsabilidade compartilhada, pois geradores de resíduos, tanto públicos como privados, tem responsailidade definida e devem cooperar para que os objetivos da PNRS sejam alcançados (PATELA, 2011; PEREIRA, 2012).

Por mais conveniente, a nova política ainda é motivo de dúvidas, debates e tema de estudo de viabilidade (seja ela econômica, técnica ou cultural) de implantação. Para lidar com tais disparidades, grupos de trabalho e acordos setoriais, tendo em vista a implantação da responsabilidade compartilhada pelo ciclo de vida do produto, foram criados entre os diversos sujeitos envolvidos a fim de implementar essa nova sistemática. Trata-se de uma responsabilidade compartilhada, onde o consumidor final é um elemento chave.

Apesar de não fazer citação direta a medicamentos, a PNRS deixa claro, conforme previsto no seu Art. 33 que prioriza produtos em função do grau e extensão do impacto à saúde pública e ao meio ambiente. Desta forma, a Figura 2 apresenta a ordem de prioridade para o gerenciamento dos resíduos sólidos estabelecida pela PNRS no seu Art. $9^{\circ}$. O prazo para implantação das medidas de disposição final, ambientalmente adequada dos rejeitos, é de quatro anos após a publicação da PNRS. 


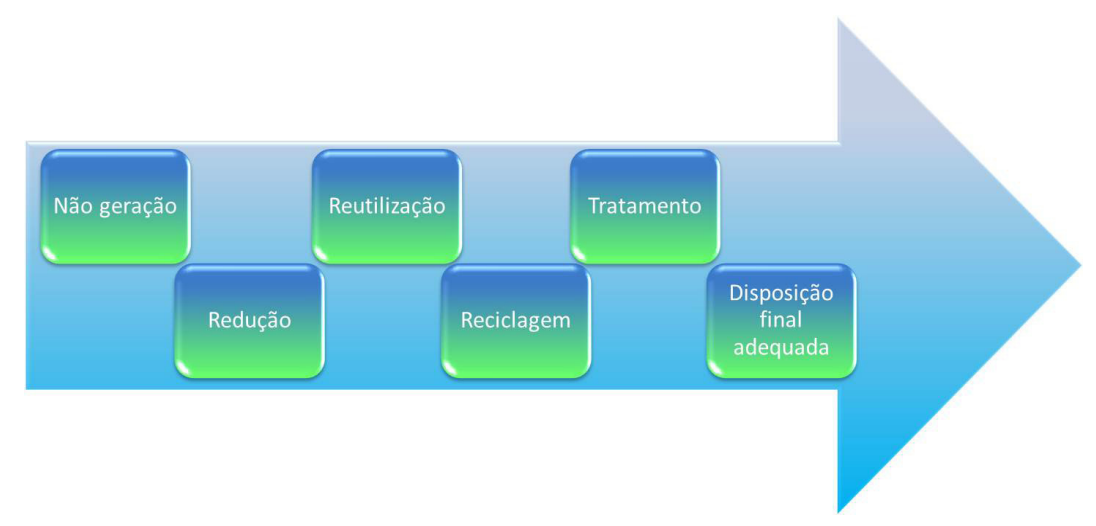

Figura 2 - Ordem De Prioridade Para Gerenciamento De Resíduos Sólidos

\section{I.4 Descarte de Medicamentos}

No Brasil são geradas cerca de 120 mil toneladas de lixo por dia, sendo que de 1 a $3 \%$ desse total é produzido por estabelecimentos de saúde e, destes, $10 \%$ a $25 \%$ representam risco ao meio ambiente e à saúde da população (ANVISA, 2006; RODRIGUES, 2009). O Brasil junto com os Estudos Unidos, França e Alemanha constituem os maiores consumidores de medicamentos do mundo (JESUS, 2004; PEDROSO, 2007).

O descarte indevido de medicamentos é uma importante causa da contaminação do meio ambiente, sendo que suas consequências ainda não são muito conhecidas (FENT, 2006; MCCLELLAN, 2010). Alguns grupos de fármacos merecem uma atenção especial. Entre eles, estão os antibióticos e os estrogênios. Os primeiros, devido ao desenvolvimento de bactérias resistentes, e os estrogênios, pelo seu potencial de afetar adversamente o sistema reprodutivo de organismos aquáticos como, por exemplo, a feminização de peixes machos presentes em rios contaminados com descarte de efluentes de estações de tratamento de esgoto (FALQUETO, 2010; JOÃO, 2011).

É sabido que a destinação adequada, ambientalmente correta, de medicamentos no Brasil ainda não está estruturada (FALQUETO, 2010; BARCELOS, 2011). Apesar de existirem iniciativas e regulamentações diversas sobre devolução, recolhimento, doação e descarte de medicamentos em desuso, muitas vezes o destino destes acaba sendo os esgotos ou o lixo comum.

Os resíduos de medicamentos são classificados como resíduos do grupo B, que engloba substâncias químicas que podem apresentar risco à saúde pública ou ao meio ambiente, dependendo de suas características de inflamabilidade, corrosividade, reatividade e toxicidade (BRASIL, 2004).

Dentre os fatores relacionados com as sobras de medicamentos destacam-se: a sua distribuição em excesso, acima das quantidades exatas para o paciente; distribuição de amostras-grátis; falta de instruções e informações da população; mudança de tratamento ou interrupção; estabelecimentos de saúde com gerência de estoque inadequada (JOÃO, 2011).

Os riscos que advêm destes inúmeros fatores variam das graves consequências nos aspectos ambientais (contaminação do solo, animais, água, etc.) até a reutilização por pessoas, proposital ou não, muitas vezes devido a questões socioculturais. O consumo indevido de medicamentos pode provocar diversos problemas como intoxicações e graves reações adversas.

\section{METODOLOGIA}

A metodologia utilizada para a concepção desta pesquisa foi através de pesquisas bibliográficas, abordando sobre o tema "logística reversa" e a legislação que envolve o descarte correto de medicamentos. Quase todos os estudos são submetidos a esse tipo de trabalho, enquanto outros são desenvolvidos unicamente por meio de fontes bibliográficas. Entre as vantagens de uso pode-se notar que são fontes ricas e estáveis de informação, e de custo relativamente baixo (DIEHL, 2004).

$\mathrm{Na}$ segunda parte do estudo realizou-se um estudo de caso que consistiu na busca por infor- 
mações sobre certo indivíduo, família, grupo ou comunidade para analisar diferentes aspectos de sua vida (CERVO, 2007). Segundo Yin (2009), este estudo é uma investigação empírica que analisa um fenômeno presente dentro de uma situação da vida real.

O estudo concentrou-se em cinco cidades da Região do Vale do Rio Pardo, localizado no centro do Estado do Rio Grande do Sul, distante $150 \mathrm{~km}$ da capital, que juntas somam uma população de 276.000 habitantes, segundo o Censo 2010. Os dados foram obtidos pela aplicação de um questionário fechado contendo cinco questões, no período de setembro a dezembro de 2012, em 50 farmácias e drogarias. Procurou-se entrevistar pessoas com o mesmo nível hierárquico e atividade funcional, de forma a uniformizar as informações de cada caso e propiciar sua replicação.

O questionário abordou as seguintes perguntas: (1) A empresa possui um programa de destinação correta de resíduos? (2) Há quanto tempo? (3) Com que frequência é feita a coleta? (4) Vocês tem conhecimento do destino que é dado ao resíduo pela empresa que o recolhe? (5) A empresa possui algum método para divulgar e/ou incentivar a população a participar do recolhimento dos medicamentos em desuso?

\section{RESULTADOS E DISCUSSÕES}

Para realização desta pesquisa, foram elaboradas algumas questões para avaliar a respeito do descarte de medicamentos e da logística reversa, estando intrínseco o assunto pelas normativas relacionadas. Das cinquenta farmácias selecionadas na região do Vale do Rio Pardo foram escolhidas as pessoas para responderem o questionário. Essas se classificavam como os responsáveis pelo estabelecimento ou os profissionais que possuíam vínculo direto, dentro do seu cargo, com a área. Para uma análise clara e organizada, os resultados encontram-se representados nos gráficos das figuras 3 a $7 \mathrm{com}$ as respectivas questões formuladas.

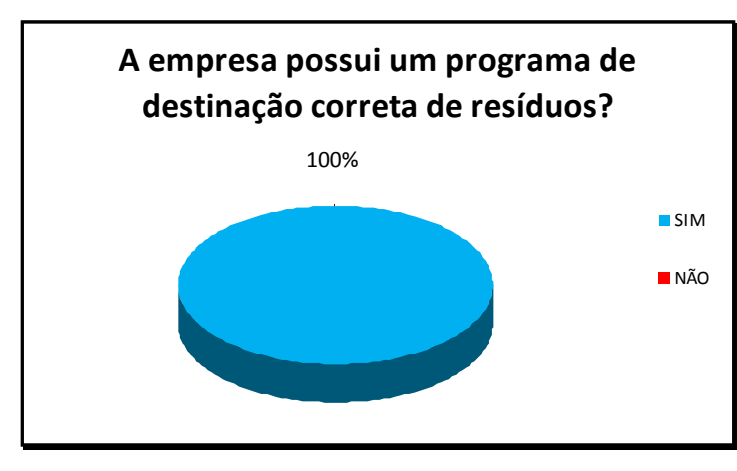

Figura 3 - Resultados Obtidos Pergunta 1

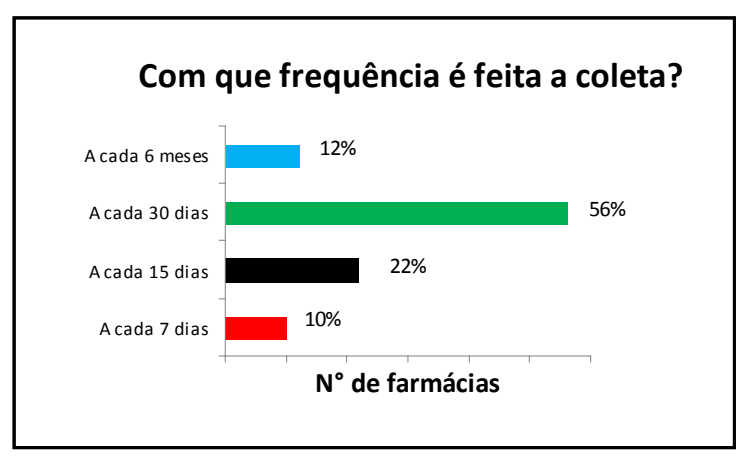

Figura 5 - Resultados Obtidos Pergunta 3

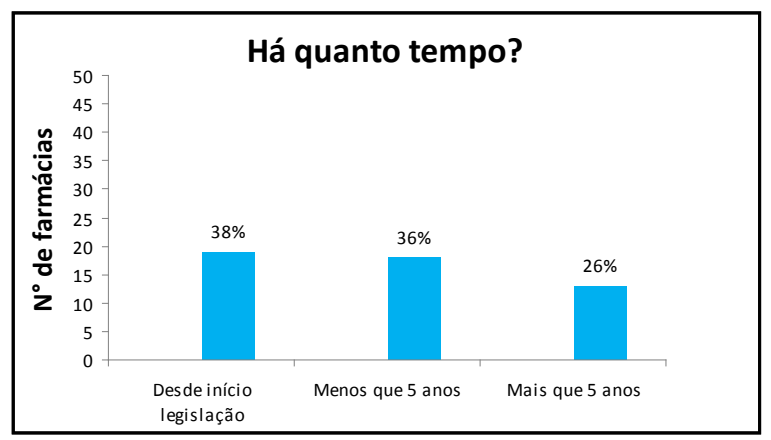

Figura 4 - Resultados Obtidos Pergunta 2



Figura 6 - Resultados Obtidos Pergunta 4 


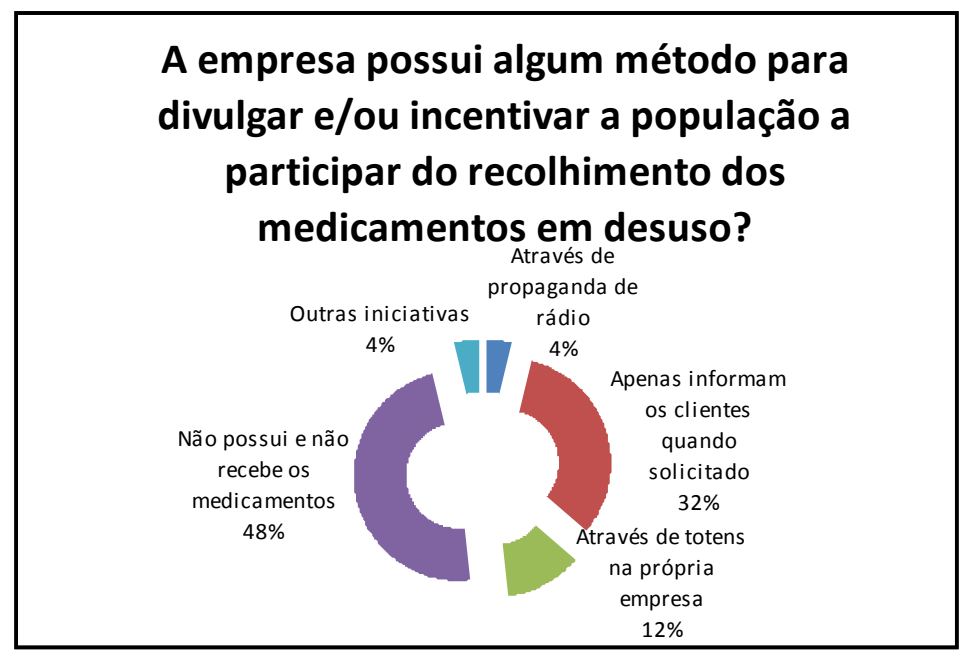

Figura 7 - Resultados Obtidos Pergunta 5.

\section{CONCLUSÕES}

A aplicação de logística reversa para medicamentos em desuso é um tema muito recente, principalmente para a sociedade em si, que carece de informação e divulgação para saber o que fazer com esses produtos.

Pode-se verificar que não é de pleno domínio das farmácias a legislação pertinente, visto que conforme os resultados e as entrevistas, apesar de todas estarem implementando um programa de destinação, poucas entendem sobre ou sabem o que acontece com os resíduos. E apenas uma pequena parcela das farmácias demonstrou iniciativas de divulgação e conscientização. Além disso, cabe a uma parcela maior aceitar os medicamentos obsoletos e vencidos da população, visto que a maioria das pesquisadas, conforme estudo, apenas recolhe seus próprios resíduos.

Apesar da redação e da obrigatoriedade de implementação da PNRS em 2010, poucos são os etabelecimentos comerciais e industriais que estão cumprindo o que determina a referida lei 12.305 . Não se deve esquecer a real importância e o foco principal da logística reversa: o menor dano ambiental. Os resíduos de medicamentos são extremamente nocivos e mal gerenciados atualmente pela população, impactando negativamente o meio ambiente se não controlados e administrados com a importância que lhe é peculiar.

\section{REFERÊNCIAS}

ANVISA, resolução no 306 de 7 de dezembro de 2004, que dispõe sobre o Regulamento Técnico para o gerenciamento de resíduos de serviços de saúde, Brasília: 2010.

BALLOU, Ronald H. Gerenciamento da cadeia de suprimentos/logística empresarial. 5. ed. Porto Alegre: Bookman, 2006.

BARCELOS, M. N.; et al. Aplicação do método FMEA na identificação de impactos ambientais causados pelo descarte doméstico de medicamentos. Revista Engenharia Ambiental: Pesquisa e Tecnologia, v. 8, n. 4, p.62-68,2011.

BOER, Noemi. Descarte de medicamentos: um modelo de logística reversa. Congresso Responsabilidade e Reciprocidade. Fundação Antonio Meneghetti \& Faculdade Antonio Meneghetti. Restinga Seca, 2011

BOWERSOX, D. J.; CLOSS, D. J. Logística empresarial: o processo da integração da cadeia de suprimento. São Paulo: Atlas, 2001. 
BRASIL, Lei N ${ }^{\circ} \mathbf{1 2 . 3 0 5}$ de 2 de Agosto de 2010, Política Nacional de Resíduos Sólidos (PNRS), Brasília: 2010.

CERVO, A. L.; BERVIAN, P. A.; SILVA, R. Metodologia Científica. 4 ed. São Paulo: Pearson Prentice Hall, 2007.

CONSELHO EM REVISTA., ANO VII | No 85. Porto Alegre: Mensal. set 2011

DIEHL, A. A.; TATIM, D. C. Pesquisa em ciências sociais aplicadas. 1 ed. São Paulo: Pearson Prentice Hall, 2004.

FALQUETO, E.; KLIGERMAN, D. C., ASSUMPCAO, R. F.i. Como realizar o correto descarte de resíduos de medicamentos? Ciência saúde coletiva. vol.15, n.2, p. 3283-3293, 2010.

FENT, K.; WESTON, A. A.; CAMINADA, D. Ecotoxicology of human pharmaceuticals. Aquatic Toxicology. v.76, p.122-159, 2006.

FERRER, G; AYRES R. The impact of remanufacturing in the economy. Ecological Economics. v. 32, n. 3, p. 413-429, 2000.

GONÇALVES, M. E.; MARINS, F. A. S. Logística reversa numa empresa de laminação de vidros: um estudo de caso. Gestão e Produção. v. 13, n. 3, p. 397-410, set./dez. 2006.

JESUS, Paula Renata C. . As palavras e seus efeitos na propaganda de medicamentos no Brasil: uma análise semiótica, midiática e ética. In: 7 Congreso Latinoamericano de Investigadores de La Comunicación, 2004, La Plata. Comunicación y Salud, 2004.

JERÔNIMO, C. E. M.; CÂMARA, S. P. As consequências sócio-ambientais decorrentes do descarte inadequado dos resíduos sólidos no município de Brejinho-RN. Rev. Elet. em Gestão, Educação e Tecnologia Ambiental. v.10, n. 10, p. 2165-2174, jan./abr. 2013

JOÃO, Walter da Silva Jorge. Descarte de medicamentos. Pharmacia Brasileira. n. 82, 2011.

KEMERICH, P. D. C.; et al. Gestão dos resíduos sólidos urbanos: a visão da população na cidade de Frederico Westphalen. Rev. Elet. em Gestão, Educação e Tecnologia Ambiental. v.10, n. 10, p. 2059-2068, jan./ abr. 2013

LAMBERT, S.; RIOPEL, D.; ABDUL-KADER, W. A reverse logistics decisions conceptual framework. Computers \& Industrial Engineering, v. 61, p. 561-581, 2011.

LEITE, P. R. Logística Reversa: Meio ambiente e competitividade. 2 ed. São Paulo: Pearson Prentice Hall, 2009.

MARGONATO, F. B.; THOMSON, Z.; PAOLIELLO, M. M. B. Determinantes nas intoxicações medicamentosas agudas na zona urbana de um município do Sul do Brasil. Caderno Saúde Pública, Rio de Janeiro, 24 (2):333-341, fev, 2008.

MCCLELLAN, K.; HALDEN, R.U. Pharmaceuticals and personal care products in archived U.S. biosolids from the 2001 EPA national sewage sludge survey. Water Research. v.44, p. 658-668, 2010.

MELO, S. A.S.;TROVÓ, A. G.; BAUTITZ, I. R.; NOGUEIRA, R. F. P. Degradação de fármacos residuais por processos oxidativos avançados. Revista Química Nova, São Paulo, vol.32, nº 1. 188-197, 2009.

MELO JÚNIOR, T. A.; DÂNDARO F.; AMBROSETO, G.; TABAH J. Estudo de caso: coleta e logística 
reversa para lâmpadas fluorescentes no município de Franca, SP. Rev. Elet. em Gestão, Educação e Tecnologia Ambiental. v.10, n. 10, p. 2091-2101, jan./abr. 2013

NOVAES, A. G. Logística e gerenciamento da cadeia de distribuição: estratégia, operação e avaliação. Rio de Janeiro: Campus, 2001.

RADONJIC, G.; TOMINC, P. The role of environmental management system on introduction of new technologies in the metal and chemical/paper/plastics industries. Journal of Cleaner Production, n. 15, p. 14821493, 2007.

RAVI, V; SHANKAR R. Analysis of interactions among the barriers of reverse logistics. Technological Forecasting and Social Change, v. 72, n. 8, p. 1011-1029, 2005.

RODRIGUES, Carla Regina Blanski. Aspectos Legais e Ambientais do Descarte de Resíduos de Medicamentos. 2009. Dissertação de mestrado em Engenharia de Produção, apresentada a Universidade Tecnológica Federal do Paraná. Ponta Grossa. 2009.

ROGERS, Dale S.; TIBBEN-LEMBKE, Ronald. An examination of reverse logistics practices. Journal of business logistics, v. 22, n. 2., 2001.

SILVA, A. L. E.; MORAES, J. A. R.; MACHADO, E. L. Proposta de um programa de P+L como ferramenta para promoção da gestão ambiental: estudo de caso. Revista Tecno-Lógica, v. 16, n. 1, p. 40-47, 2012.

SINNECKER, Carlos Alberto. Estudo sobre a importância da Logística Reversa em quatro grandes empresas da região metropolitana de Curitiba. 2007. Dissertação (Programa Pós-Graduação em Engenharia de Produção e Sistemas - PPGEPS) - Pontifícia Universidade Católica do Paraná, Curitiba, 2007.

SRIVASTAVA, Samir K.. Network design for reverse logistics. The International Jounal of Management Science, v. 36, p. 535-548, 2008.

STOCK, James R. Reverse logistics in the supply chain. Transport \& Logistics, 2002

PATELA, Luciana. Responsabilidade compartilhada, acordos setoriais e logística reversa: a PNRS na prática. Revista do Crea, ano 7, n. 85, p. 16-18, 2011.

PEDROSO, C.F. Indústria farmacêutica. Acta Farmaceutica Bonaerense, v.26, 2007.

PEREIRA, S. S.; CURI, R. C. Modelos de gestão integrada dos resíduos sólidos urbanos: a importância dos catadores de materiais recicláveis no processo de gestão ambiental. Revista Engenharia Ambiental: Pesquisa e Tecnologia, v. 9, n. 4, p. 118-138, 2012.

POKHAREL, S.; MUTHA, A. Perspectives in reverse logistics: a review. Resources, Conservation and Recycling, v. 53, n. 4, p. 175-182, 2009.

YIN, R. K. Case Study Research - Design and Methods. 4 ed. USA: Sage Publications Inc., 2009.

ZHANG, Y. M.; et al. An inexact reverse logistics model for municipal solid waste management systems.

Journal of Environmental Management. v.92, p.522-530, 2011. 